\title{
Niveles de Monóxido de Carbono causado por la festividad de "San Juan" en la ciudad de Pucallpa 2021
}

\section{Carbon Monoxide levels caused by the festival of "San Juan" in the city of Pucallpa 2021}

\author{
Paul Kevin Reátegui Ramos ${ }^{1}$, Isabel Esteban Robladillo ${ }^{1}$, Dina Parí Quispe ${ }^{1}$, Jenny Paola Zeña Rubio ${ }^{1}$, Pablo \\ Caruzo Sangama ${ }^{1}$, Susy Bernal Apaestegui ${ }^{1}$
}

${ }^{1}$ Universidad Nacional de Ucayali. Carretera Federico Basadre km 6.200. Ucayali, Perú. Correo electrónico: paulreateguiramos@gmail.com. ORCID: https://orcid.org/0000-0002-9916-8107

${ }^{1}$ Universidad Nacional de Ucayali. Carretera Federico Basadre km 6.200. Ucayali, Perú. Correo electrónico: isabel_esteban@unu.edu.pe. ORCID: https://orcid.org/0000-0002-7523-6654

${ }^{1}$ Universidad Nacional de Ucayali. Carretera Federico Basadre km 6.200. Ucayali, Perú. Correo electrónico: dina_pari@unu.edu.pe. ORCID: https://orcid.org/0000-0002-1493-9209

${ }^{1}$ Universidad Nacional de Ucayali. Carretera Federico Basadre km 6.200. Ucayali, Perú. Correo electrónico: jenny_zeña@unu.edu.pe.

\section{Resumen}

El presente estudio tuvo como objetivo evaluar los Niveles de Monóxido de Carbono causado por la festividad de "San Juan" en la ciudad de Pucallpa 2021. La metodología utilizada fue mediante el uso de sensores electroquímicos, tomando 2 horarios específicos diurno ( 7 am a 2 pm) y nocturno (6 pm a $1 \mathrm{am}$ ) en rangos por punto de muestro con intervalos de análisis de 5 segundos para la obtención del promedio. Se analizaron 12 puntos distribuidos aleatoriamente en la ciudad de Pucallpa, evaluados por 9 días. Los resultados indican que 8 de 10 familias usaron leña o carbón para la cocción del Juane y que 9 de 10 familias elaboraron o cocinaron el juane de la fiesta de "San Juan- Pucallpa 2021" el 23 de junio (un día antes de la Fiesta). Así mismo los niveles de Monóxido de Carbono antes de la festividad de "San Juan" fueron en promedio de $4.270 \mathrm{mg} / \mathrm{m} 3$, durante promedio $6.660 \mathrm{mg} / \mathrm{m} 3 \mathrm{y}$ después promedio de $4.940 \mathrm{mg} / \mathrm{m} 3$ en la ciudad de Pucallpa en el año 2021. Se concluye que el efecto en los niveles de Monóxido de Carbono causado por la festividad de "San Juan" en la ciudad de Pucallpa en el 2021, es que los Índices de Nacionales de Calidad de Aire fueron "Buenos" antes y después de la festividad, pero de clasificación "Moderada" durante esta.

Palabras clave: Monóxido de Carbono, Fiesta de San Juan, Calidad de Aire.

\begin{abstract}
The objective of this study was to evaluate the Carbon Monoxide Levels caused by the "San Juan" festival in the city of Pucallpa 2021. The methodology used was through the use of electrochemical sensors, taking 2 schedules specific diurnal (7 am to $2 \mathrm{pm})$ and night (6 pm to $1 \mathrm{am})$ in ranges per sampling point with analysis intervals of 5 seconds to obtain the average. Twelve randomly distributed points in the city of Pucallpa were analyzed, evaluated for 9 days. The results indicate that 8 out of 10 families used firewood or charcoal to cook the Juane and that 9 out of 10 families made or cooked the juane for the festival of "San Juan- Pucallpa 2021" on June 23 (one day before the festival). Likewise, the levels of Carbon Monoxide before the festival of "San Juan" were an average of 4,270 mg / m3, during an average $6,660 \mathrm{mg} / \mathrm{m} 3$ and then an average of 4,940 $\mathrm{mg} / \mathrm{m} 3$ in the city of Pucallpa in the year 2021. It is concluded that the effect on the levels of Carbon Monoxide caused

$$
615
$$

Downloadable from: http://revistas.unu.edu.pe

Carretera Federico Basadre $\mathrm{Km}$ 6, Dirección de Producción Intelectual
\end{abstract}

Revista de Investigación Universitaria por Universidad Nacional de Ucayali se distribuye bajo una Licencia Creative Commons Atribución-NoComercial 4.0 Internacional. 
by the festival of "San Juan" in the city of Pucallpa in 2021, is that the National Air Quality Indices were "Good" before and after the festival, but classified as "Moderate" during this.

Keywords: Carbon Monoxide, Fiesta de San Juan, Air Quality.

\section{Introducción}

El 18 de marzo del 2010, mediante la Resolución Directoral Nacional N575-INC, se declara patrimonio cultural de la nación a la "Fiesta de San Juan" de la Amazonia peruana, en, celebrada el 24 de junio según el calendario cristiano católico (Instituto Nacional de Cultura, 2010).

La Fiesta de San Juan es la celebración más importante y desbordante de los pueblos de la Amazonía peruana. Definitivamente, el plato fuerte y símbolo de esta fecha es el popular juane, una mezcla de arroz, huevos cocidos, gallina y especias envueltas en una hoja de bijao, la cual simboliza la cabeza de San Juan Bautista y cocinarla a leña tradicionalmente. Es normal ver a distintas amas de casa el 23 de junio levantarse a horas de la madrugada para asistir al mercado en busca de los insumos para preparar la emblemática comida (ANDINA, 2019) (Canepa Koch y otros, 2011). La Fiesta de San Juan y como es costumbre, el plato estrella y que no falta en ninguna mesa es el juane (Perú21, 2017).

Alrededor del $40 \%$ de la población mundial sigue utilizando combustibles sólidos, entre ellos la leña, para cocinar o calentar sus hogares (Torres y otros, 2016) (Smith, 2006). La forma común de la cocción es mediante leña y carbón. Según Public Health Madison \& Dane County (2004), los artefactos de calefacción a leña (estufas, calderas u hornos) cada vez que se encienden pueden producir humo denso durante horas. Independientemente de que el humo se vea o no, contiene: PM 2.5, Monóxido de Carbono, Ozono, Óxido Nitroso, Dióxido de Azufre e Hidrocarburos Aromáticos Policiclicos. Los gases tóxicos y las diminutas partículas del humo de la combustión de leña son pequeños. El humo de la leña que no se quema por completo contiene una cantidad de sustancias químicas que incluye monóxido de carbono (CO). (EPA, 2021).

Las probabilidades de sufrir consecuencias en la salud por la exposición al humo dependen de la concentración de contaminantes en el aire y del tiempo que haya estado expuesto a ellos. Respirar el humo de la combustión de leña es un riesgo para la salud. Los niños, los ancianos, y las personas con asma, enfermedades del corazón y otras enfermedades respiratorias están a mayor 
UNIVERSIDAD NACIONAL DE UCAYALI

Revista de Investigación Universitaria

Versión electrónica 2664 - 8423

ARTICULO ORIGINAL
Vol. $11 \mathrm{~N}^{\circ}$ 2, pp. $615-629$, julio/diciembre 2021

Recibido 02/10/2021

Aceptado 18/12/2021

Publicado 30/12/2021 riesgo (Public Health Madison \& Dane County, 2004) (Pérez y otros, 1999).

Alrededor de 3 billones de personas en el mundo cocinan sus alimentos en sus hogares, utilizando fuego abierto y cocinas tradicionales de quema de biomasa (leña, residuos animales o vegetales). En los últimos años, el tema del impacto del uso de cocinas a leña en países en desarrollo ha recibido una gran atención debido a su directa relación con aspectos ambientales, de salud y socioeconómicos (Nige y otros, 2000)

\section{Metodología}

\section{Población y muestra}

La población estuvo conformada por el área urbana de la ciudad de Pucallpa que comprende los distritos de Calleria, Manantay y Yarinacocha.

Tabla 1

Ubicación de puntos de monitoreo de Monóxido de Carbono en la ciudad de Pucallpa

\begin{tabular}{|c|c|c|c|c|c|}
\hline \multirow[t]{2}{*}{ Ítem } & \multirow{2}{*}{ Distrito } & \multirow{2}{*}{ Ubicación } & \multicolumn{2}{|c|}{ Coordenada } & \multirow{2}{*}{$\begin{array}{c}\text { Altura } \\
\text { m.S.n.m. }\end{array}$} \\
\hline & & & $\mathrm{X}$ & $\mathrm{Y}$ & \\
\hline 1 & Calleria & $\begin{array}{lrr}\text { Av. Centenario } & / & \text { Av. } \\
\text { Habilitación } & \text { Urbana } \\
\text { Municipal } & \end{array}$ & $545954.47 \mathrm{~m} \mathrm{E}$ & $9072234.74 \mathrm{~m} \mathrm{~S}$ & 157 \\
\hline 2 & Calleria & Av. Sáenz Peña / Jr. Tarapacá & $551023.10 \mathrm{~m} \mathrm{E}$ & $9073718.26 \mathrm{~m} \mathrm{~S}$ & 156 \\
\hline 3 & Calleria & Av. Miraflores / Jr. Arenales & $550460.35 \mathrm{~m} \mathrm{E}$ & $9075110.19 \mathrm{~m} \mathrm{~S}$ & 150 \\
\hline 4 & Calleria & $\begin{array}{l}\text { Av. Unión / Av. Salvador } \\
\text { Allende }\end{array}$ & $549510.65 \mathrm{~m} \mathrm{E}$ & $9073793.76 \mathrm{~m} \mathrm{~S}$ & 149 \\
\hline 5 & Manantay & $\begin{array}{l}\text { Av. Túpac Amaru II / Av. } \\
\text { Aviación }\end{array}$ & $548140.20 \mathrm{~m} \mathrm{E}$ & $9071637.83 \mathrm{~m} \mathrm{~S}$ & 151 \\
\hline 6 & Manantay & $\begin{array}{l}\text { Jr. Pachacutec / } \\
\text { Eucaliptos (Plaza } \\
\text { Bozo) }\end{array}$ & $548214.99 \mathrm{~m} \mathrm{E}$ & $9070420.01 \mathrm{~m} \mathrm{~S}$ & 156 \\
\hline 7 & Manantay & $\begin{array}{l}\text { Jr. Los Laureles / Av. } \\
\text { Colonización }\end{array}$ & $549754.56 \mathrm{~m} \mathrm{E}$ & $9070983.35 \mathrm{~m} \mathrm{~S}$ & 158 \\
\hline 8 & Manantay & $\begin{array}{l}\text { Av. Túpac Amaru II / Jr. } \\
\text { Aguaytia (Mun. Manantay) }\end{array}$ & $550557.55 \mathrm{~m} \mathrm{E}$ & $9071413.25 \mathrm{~m} \mathrm{~S}$ & 155 \\
\hline 9 & Yarinacocha & $\begin{array}{l}\text { Av. Miraflores / Av. } \\
\text { Yarinacocha }\end{array}$ & $548893.57 \mathrm{~m} \mathrm{E}$ & $9074635.72 \mathrm{~m} \mathrm{~S}$ & 154 \\
\hline 10 & Yarinacocha & $\begin{array}{l}\text { Av. Yarinacocha / Jr. Nueva } \\
\text { Luz de Fátima }\end{array}$ & $547055.71 \mathrm{~m} \mathrm{E}$ & $9076041.13 \mathrm{~m} \mathrm{~S}$ & 155 \\
\hline 11 & Yarinacocha & Av. Arborización / Av. Unión & $547405.62 \mathrm{~m} \mathrm{E}$ & $9073322.61 \mathrm{~m} \mathrm{~S}$ & 152 \\
\hline 12 & Yarinacocha & $\begin{array}{l}\text { Jr. Palmeras / Jr. Iquitos } \\
\text { (Plaza las Palmeras) }\end{array}$ & $546369.22 \mathrm{~m} \mathrm{E}$ & $9075519.27 \mathrm{~m} \mathrm{~S}$ & 154 \\
\hline
\end{tabular}

617

Downloadable from: http://revistas.unu.edu.pe

Carretera Federico Basadre Km 6, Dirección de Producción Intelectual

Revista de Investigación Universitaria por Universidad Nacional de Ucayali se distribuye bajo una Licencia Creative Commons Atribución-NoComercial 4.0 Internacional. 
La muestra estuvo constituida por 12 puntos distribuidos aleatoriamente en la ciudad de Pucallpa, evaluados por 9 días. Según el MINAM (2019), en el Protocolo Nacional de Monitoreo de la Calidad Ambiental del Aire; el número mínimo de estaciones de monitoreo o puntos de monitoreo de calidad de aire según el criterio poblacional, para una población de más de 6000 mill/ Hab. el número mínimo es de 10 muestras.

\section{Análisis de Monóxido de Carbono por sensores electroquímicos}

Este mecanismo de determinación de un haz "referencia" y un haz "medición", de manera alternada, incorpora un filtro de Correlación de Gas (GFC), el cual cuenta con un elemento rotatorio filtrante de gas, dividido en dos secciones, una que contiene el Monóxido de Carbono y otra que presenta Nitrógeno. El periodo o tiempo de monitoreo del gas de Monóxido de Carbono es de 8 horas, con mínima suficientes de información validad requerida del 90\% o 7 horas (MINAM, 2019).

\section{Índice de Calidad de Aire}

El índice de Calidad del Aire (INCA) tiene un valor óptimo comprendido entre O y 100, el cual coincide con el cumplimiento de los Estándares de Calidad Ambiental de Aire. El
INCA se divide en 4 categorías o calificaciones de la calidad del aire. La banda de color verde comprende valores del INCA de $\mathrm{O}$ a 50 y significa que la calidad del aire es buena, la banda de color amarillo comprende valores de 51 a 100 e indica una calidad moderada del aire; la banda de color anaranjado se encuentra comprendida entre los valores 101 y el valor umbral del estado de cuidado (VUEC) de cada contaminante, lo que nos indica que la calidad del aire es mala; finalmente el color rojo de la cuarta banda nos indica que la calidad del aire es mayor al valor umbral del estado de cuidado del contaminante (MINAM, 2016).

Formula:

$$
\mathrm{I}(\mathrm{CO})=\left(\mathrm{COug} / \mathrm{M}^{3}\right) * 100 / 10000
$$

-COug/ $\mathrm{M}^{3}=$ Concentración del contamínate

$-10000=$ Valor del estándar del ECA Aire para Monóxido de Carbono 
UNIVERSIDAD NACIONAL DE UCAYALI

Revista de Investigación Universitaria

Versión electrónica 2664 - 8423

ARTICULO ORIGINAL
Vol. $11 \mathrm{~N}^{\circ}$ 2, pp. 615 - 629, julio/diciembre 2021

Recibido 02/10/2021

Aceptado 18/12/2021

Publicado 30/12/2021

\section{Ubicación de los puntos de monitoreo}

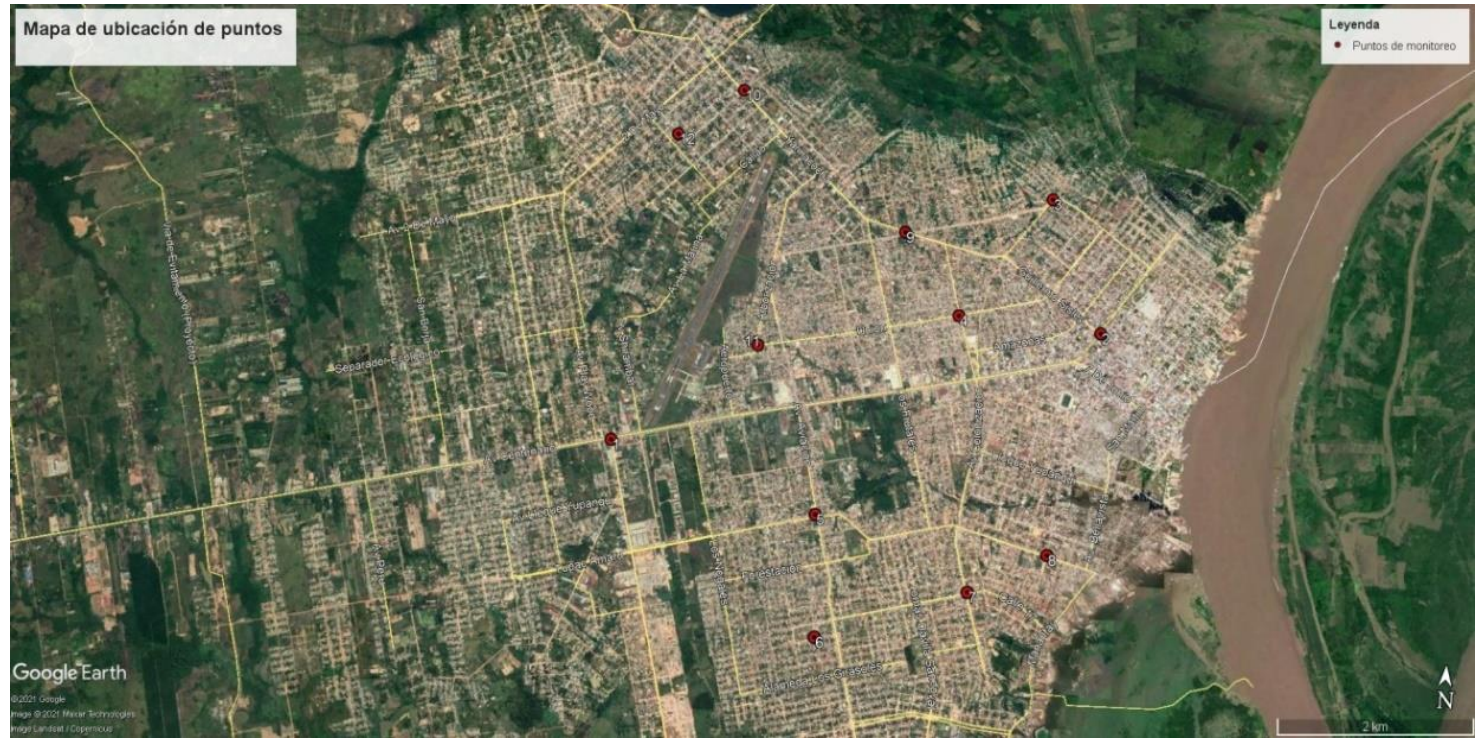

Figura 1: Mapa de ubicación de puntos

\section{Resultados y Discusión}

Tabla 2

Uso de leña o carbón para la cocción de juane en la fiesta de "San Juan"- Pucallpa 2021

\begin{tabular}{lllll}
\hline Distrito & N & Carbón & Leña & Gas \\
\hline Calleria & 70 & 3 & 51 & 16 \\
Manantay & 65 & 12 & 44 & 9 \\
Yarinacocha & 68 & 17 & 46 & 5 \\
\hline
\end{tabular}

El uso de leña o carbón para la cocción de juane en la fiesta de "San Juan"- Pucallpa 2021, en el distrito de Calleria el $77 \%$ de la población uso leña y carbón solo el $23 \%$ uso gas, en el distrito de Manantay el $86 \%$ de la población uso leña y carbón solo el 14\% uso gas y en el distrito de Yarinacocha el 93\% de la población uso leña y carbón solo el $7 \%$ uso gas. Se puede indicar que 8 de 10 familias usaron leña o carbón para la cocción del Juane en la fiesta de "San Juan"- Pucallpa 2021. 
Tabla 3

Fecha de elaboración o cocción del juane en la fiesta de "San Juan"- Pucallpa 2021

\begin{tabular}{lcccccc}
\hline \multicolumn{1}{c}{ Distrito } & N & $\mathbf{2 2} *$ & $\mathbf{2 3}^{*}$ & $\mathbf{2 4}^{*}$ & $\mathbf{2 5}^{*}$ & $\mathbf{2 6}^{*}$ \\
\hline Calleria & 70 & 1 & 65 & 4 & 0 & 0 \\
Manantay & 65 & 2 & 56 & 7 & 0 & 0 \\
Yarinacocha & 68 & 1 & 60 & 6 & 1 & 0 \\
\hline
\end{tabular}

La Fecha de elaboración o cocción del juane en la fiesta de "San Juan"- Pucallpa 2021, en el distrito de Calleria el 93\% de la población elabora el juanes del día 23 de junio (un día antes de la Fiesta), el 6\% el 24 de junio (día de la Fiesta) y el $1 \%$ el 22 de junio (dos días antes de la Fiesta); en el distrito de Manantay el 86\% de la población elabora el juanes del día 23 de junio (un día antes de la Fiesta), el $11 \%$ el 24 de junio (día de la Fiesta) y el 3\% el 22 de junio (dos días antes de la Fiesta) y en el distrito de Yarinacocha el $88 \%$ de la población elabora el juanes del día 23 de junio (un día antes de la Fiesta), el 9\% el 24 de junio (día de la Fiesta), el $1 \%$ el 22 de junio (dos días antes de la Fiesta) y el $1 \%$ el 25 de junio (un día después de la Fiesta). Se puede indicar que 9 de 10 familias elaboraron o cocinaron el juane de la fiesta de "San Juan- Pucallpa 2021" el 23 de junio (un día antes de la Fiesta). Las familias que elaboraron con dos días antes y un día después, indican que lo hicieron excepcionalmente para enviar los juanes a su familia fuera de la región. En muchos casos esta cocción por conocimiento ancestral lo realizan en un área abierta y cumpliendo con este criterio algunas familias llevan a cabo la cocción en la vía pública, el cual es un caso común durante la festividad de la "Fiesta de San Juan" en la ciudad de Pucallpa.

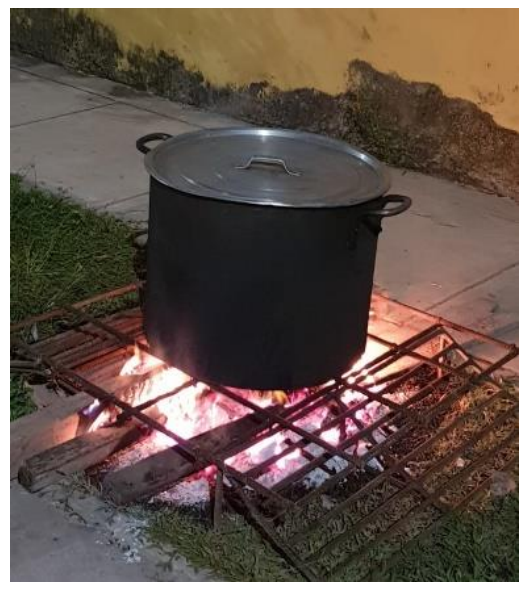

Figura 2: Cocción del preparado de "Juane de Arroz" en la vía pública de la ciudad de Pucallpa 
UNIVERSIDAD NACIONAL DE UCAYALI

Revista de Investigación Universitaria

Versión electrónica 2664 - 8423

ARTICULO ORIGINAL
Vol. $11 \mathrm{~N}^{\circ} 2$, pp. $615-629$, julio/diciembre 2021

Recibido 02/10/2021

Aceptado 18/12/2021

Publicado 30/12/2021

Tabla 4

Resultados generales del monitoreo de Monóxido de Carbono en la ciudad de Pucallpa por Fiestas de "San Juan"

\begin{tabular}{|c|c|c|c|c|c|c|c|c|c|c|c|}
\hline \multirow{2}{*}{ Ítem } & \multirow{2}{*}{ Distrito } & \multirow{2}{*}{ Ubicación /Días } & \multicolumn{3}{|c|}{ Antes } & \multicolumn{3}{|c|}{ Durante } & \multicolumn{3}{|c|}{ Después } \\
\hline & & & 20 & 21 & 22 & 23 & 24 & 25 & 26 & 27 & 28 \\
\hline 1 & Calleria & $\begin{array}{l}\text { Av. Centenario / } \\
\text { Av. Habilitación } \\
\text { Urbana } \\
\text { Municipal }\end{array}$ & 3.5 & 6.48 & 7.5 & 10.6 & 7.1 & 7.3 & 4.8 & 2.05 & 3.65 \\
\hline 2 & Calleria & $\begin{array}{l}\text { Av. Sáenz Peña / } \\
\text { Jr. Tarapacá }\end{array}$ & 1.25 & 4.21 & 0.45 & 5.63 & 4.73 & 7.5 & 1.5 & 1.1 & 5.8 \\
\hline 3 & Calleria & $\begin{array}{c}\text { Av. Miraflores / } \\
\text { Jr. Arenales }\end{array}$ & $<0.25$ & 0.45 & 0.6 & 4.33 & 0.38 & 0.73 & 2.2 & 0.55 & 2.45 \\
\hline 4 & Calleria & $\begin{array}{l}\text { Av. Unión / Av. } \\
\text { Salvador Allende } \\
\text { Av. Túpac }\end{array}$ & 1.55 & 1.1 & 2.35 & 2.78 & 1.33 & 5.33 & 1.45 & 7.45 & 1.1 \\
\hline 5 & Manantay & $\begin{array}{c}\text { Amaru II / Av. } \\
\text { Aviación }\end{array}$ & 3.4 & 2.0 & 6.4 & 12.1 & 2.28 & 10.65 & 4.75 & 3.1 & 3.05 \\
\hline 6 & Manantay & $\begin{array}{l}\text { Jr. Pachacutec / } \\
\text { Calle. Eucaliptos } \\
\text { (Plaza Laura } \\
\text { Bozo) }\end{array}$ & 1.2 & 3.3 & 5.4 & 6.73 & 4.63 & 1.53 & 2.18 & 1.65 & 9.9 \\
\hline 7 & Manantay & $\begin{array}{c}\text { Jr. Los Laureles / } \\
\text { Av. Colonización } \\
\text { Av. Túpac }\end{array}$ & 3.1 & 2.1 & 4.6 & $\begin{array}{c}12.3 \\
3\end{array}$ & 5.75 & 4.95 & 2.95 & 4 & 4.95 \\
\hline 8 & Manantay & $\begin{array}{c}\text { Amaru II / Jr. } \\
\text { Aguaytia (Mun. } \\
\text { Manantay) }\end{array}$ & 2.98 & 3.65 & 3.33 & $\begin{array}{c}11.3 \\
3\end{array}$ & 5.95 & 6.95 & 3.05 & 6.3 & 3.8 \\
\hline 9 & Yarinacocha & $\begin{array}{l}\text { Av. Miraflores / } \\
\text { Av. Yarinacocha } \\
\text { Av. Yarinacocha }\end{array}$ & 3.9 & 4.9 & 5.4 & 12.4 & 3.35 & 5.65 & 5.5 & 1.1 & 3.95 \\
\hline 10 & Yarinacocha & $\begin{array}{c}\text { / Jr. Nueva Luz } \\
\text { de Fátima }\end{array}$ & 0.95 & 1.1 & 2.6 & 6.65 & 0.38 & 0.43 & 0.45 & 2.2 & 1.9 \\
\hline 11 & Yarinacocha & $\begin{array}{l}\text { Av. Arborización } \\
\text { / Av. Unión }\end{array}$ & 3.8 & 4.6 & 2.5 & 3.35 & 1.25 & 5.93 & 1.65 & 4.6 & 3.9 \\
\hline 12 & Yarinacocha & $\begin{array}{c}\text { Jr. Palmeras / Jr. } \\
\text { Iquitos (Plaza las } \\
\text { Palmeras) }\end{array}$ & 0.45 & 1 & 1.3 & 4.93 & 0.38 & $<0.25$ & $<0.25$ & 2.05 & $<0.25$ \\
\hline \multicolumn{3}{|c|}{ Promedio diario } & 3.56 & 4.30 & 4.96 & 8.94 & 4.73 & 6.32 & 4.36 & 4.86 & 5.59 \\
\hline \multicolumn{3}{|c|}{ Promedio de etapa } & \multicolumn{3}{|c|}{4.27} & \multicolumn{3}{|c|}{6.66} & \multicolumn{3}{|c|}{4.94} \\
\hline \multirow{2}{*}{\multicolumn{3}{|c|}{ INCA }} & \multirow{2}{*}{\multicolumn{3}{|c|}{$\begin{array}{l}42.7 \\
\text { Buena }\end{array}$}} & \multirow{2}{*}{\multicolumn{3}{|c|}{66.6}} & \multicolumn{3}{|c|}{49.4} \\
\hline & & & & & & & & & & Buena & \\
\hline
\end{tabular}

*Las unidades están expresadas en $\mathrm{mg} / \mathrm{m}^{3}$

Downloadable from: http://revistas.unu.edu.pe

Carretera Federico Basadre $\mathrm{Km}$ 6, Dirección de Producción Intelectual

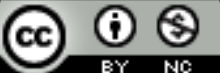

Revista de Investigación Universitaria por Universidad Nacional de Ucayali se distribuye bajo una Licencia Creative Commons Atribución-NoComercial 4.0 Internacional. 
En la tabla 4, se aprecian los niveles de Monóxido de Carbono en la ciudad de Pucallpa, antes, durante y después de las actividades de la Festividad de "San Juan", los niveles de CO "Antes" de promedian 4.270 $\mathrm{mg} / \mathrm{m}^{3}$ calificando según los INCA como Buena, "Durante" las actividades los niveles de $\mathrm{CO}$ promedian $6.66 \mathrm{mg} / \mathrm{m}^{3}$ calificando según los INCA como Moderada y los niveles de CO "Después" de promedian $4.270 \mathrm{mg} / \mathrm{m}^{3}$ calificando según los INCA como Buena. Existiendo una evidente variación de los niveles de Monóxido de Carbono influenciada por las actividades de la Festividad de "San Juan”. Así mismo podemos indicar que el distrito de Manantay es el que presenta los mayores niveles promedios de $\mathrm{CO}$ con un valor de $4.897 \mathrm{mg} / \mathrm{m}^{3}$, seguido por el distrito de Calleria con un valor de $3.376 \mathrm{mg} / \mathrm{m}^{3}$ y el distrito de Yarinacocha con un valor de 2.923 $\mathrm{mg} / \mathrm{m}^{3}$.

Los valores obtenidos no difieren en gran magnitud a los evaluados por Gomez y
Gonzalez (2020), donde la concentración encontrada de Monóxido de Carbono en la ciudad Pucallpa se promedia en 6822.13 $\mu \mathrm{g} / \mathrm{m}^{3}$, este resulta del año 2019 donde las actividades de la población se llevaban con total normalidad, se puede apreciar que también existe una diferencia con respecto a los niveles $\mathrm{CO}$ antes y después de la pandemia del COVID-19 en la ciudad de Pucallpa. Así mismo el estudio realizado por Fonseca Adianzen (2018), indica que en la ciudad de Tingo María los niveles de CO van de 277 $6023 \mu \mathrm{g} / \mathrm{m}^{3}$, del cual depende la zona de estudio, condición climática y tránsito vehicular. Así mismo el estudio realizado por Canales Manchuria (2019), indica que el promedio de registrado de $\mathrm{CO}$ en la ciudad de Arequipa es de $4453.5 \mu \mathrm{g} / \mathrm{m}^{3}$. En la ciudad de Puerto Maldonado el estudio de Bautista Baca (2018), obtuvo como resultados unos niveles de CO de 1672 a $3148 \mu \mathrm{g} / \mathrm{m}^{3}$. En diferentes ciudades del país se registran niveles $\mathrm{CO}$ en rangos cercanos a lo evaluado antes y después de la Fiesta de San Juan. 


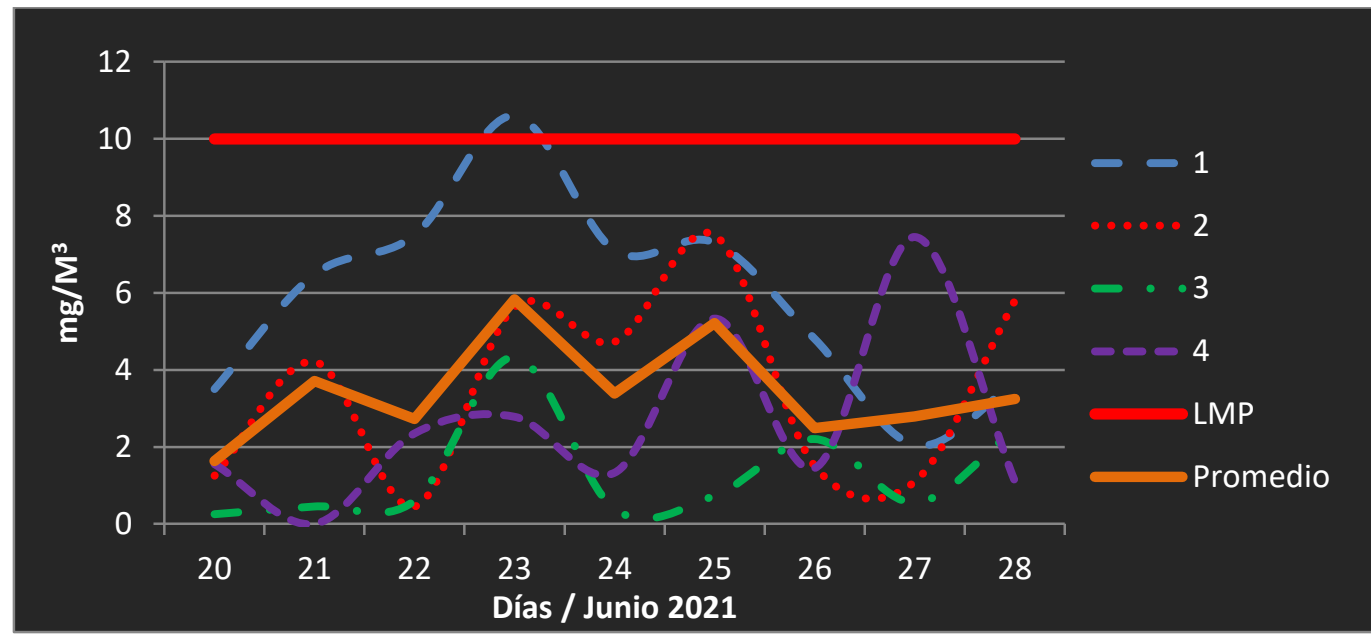

Figura 3: Comportamiento de los Niveles de Monóxido de Carbono en el distrito de Calleria por la Fiesta de San Juan 2021

Según la EPA (2020), los niveles promedio de $\mathrm{CO}$ en las casas sin el funcionamiento de estufas es de 0.5 a 5 ppm. Los niveles cercanos a las estufas de gas ajustados correctamente suelen ser de 5 a 15 ppm y aquellos cerca de estufas poco ajustadas pueden ser de $30 \mathrm{ppm}$ o más. El uso de un generador o el uso de parrillas a gas o a carbón en el interior de una vivienda sin ventilación adecuada puede producir niveles de monóxido de carbono peligrosos (ATSDR, 2012).

El estudio de Gorritty y otros (2011), indica que la tasa de emisión de concinas mejoradas a leña emiten $0.28+/-0.1 \mathrm{gCO} / \mathrm{min}$ para pruebas en 1 hora y una concentración de emisión en de 7 a 40 ppm, los cual viene a ser significativo aportante a las concentraciones de $\mathrm{CO}$ ambientales durante esta fecha ya que si se aproxima que 8 de cada 10 familias usan leña es evidente el impacto que generaría en la calidad del aire en la ciudad de Pucallpa.

En la Figura 3, se aprecia el comportamiento de los Niveles de Monóxido de Carbono en el distrito de Calleria por la Fiesta de San Juan 2021, los niveles máximos promedios de 5.835 $\mathrm{mg} / \mathrm{m}^{3} \mathrm{CO}$ fueron obtenidos durante el día 23 de Junio, el pico máximo se analizó en el punto perteneciente al cruce Av. Centenario y Av. Habilitación Urbana Municipal con un nivel de $10.6 \mathrm{mg} / \mathrm{m}^{3} \mathrm{CO}$ superando el LMP de 10 $\mathrm{mg} / \mathrm{m}^{3} \mathrm{CO}$ considerado en los Estándares de Calidad Ambiental para Aire del D.S.0032017-MINAM. 


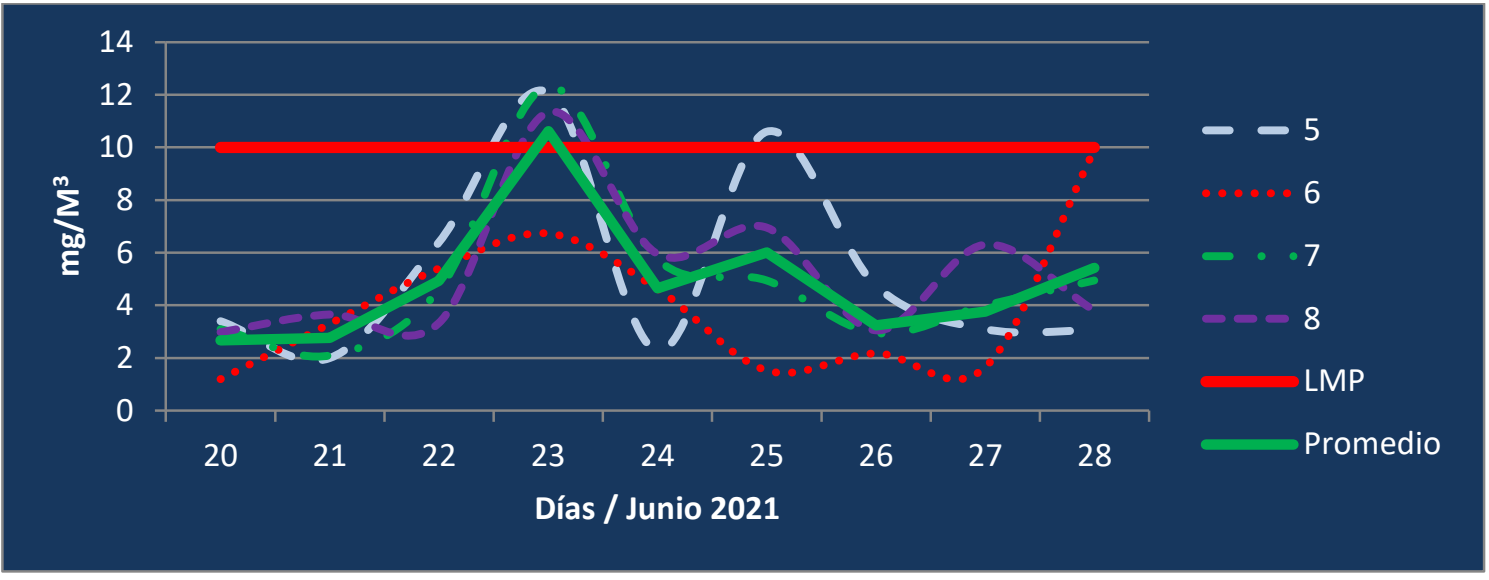

Figura 4: Comportamiento de los Niveles de Monóxido de Carbono en el distrito de Manantay por la Fiesta de San Juan 2021

En la Figura 4, se aprecia el comportamiento de los Niveles de Monóxido de Carbono en el distrito de Manantay por la Fiesta de San Juan 2021, los niveles máximos promedios de $10.623 \mathrm{mg} / \mathrm{m}^{3} \mathrm{CO}$ fueron obtenidos durante el día 23 de Junio, el pico máximo se analizó en el punto perteneciente al cruce Jr. Los Laureles y Av. Colonización con un nivel de 12.33 $\mathrm{mg} / \mathrm{m}^{3} \mathrm{CO}$ superando el LMP de $10 \mathrm{mg} / \mathrm{m}^{3} \mathrm{CO}$ considerado en los Estándares de Calidad Ambiental para Aire del D.S.003-2017MINAM.

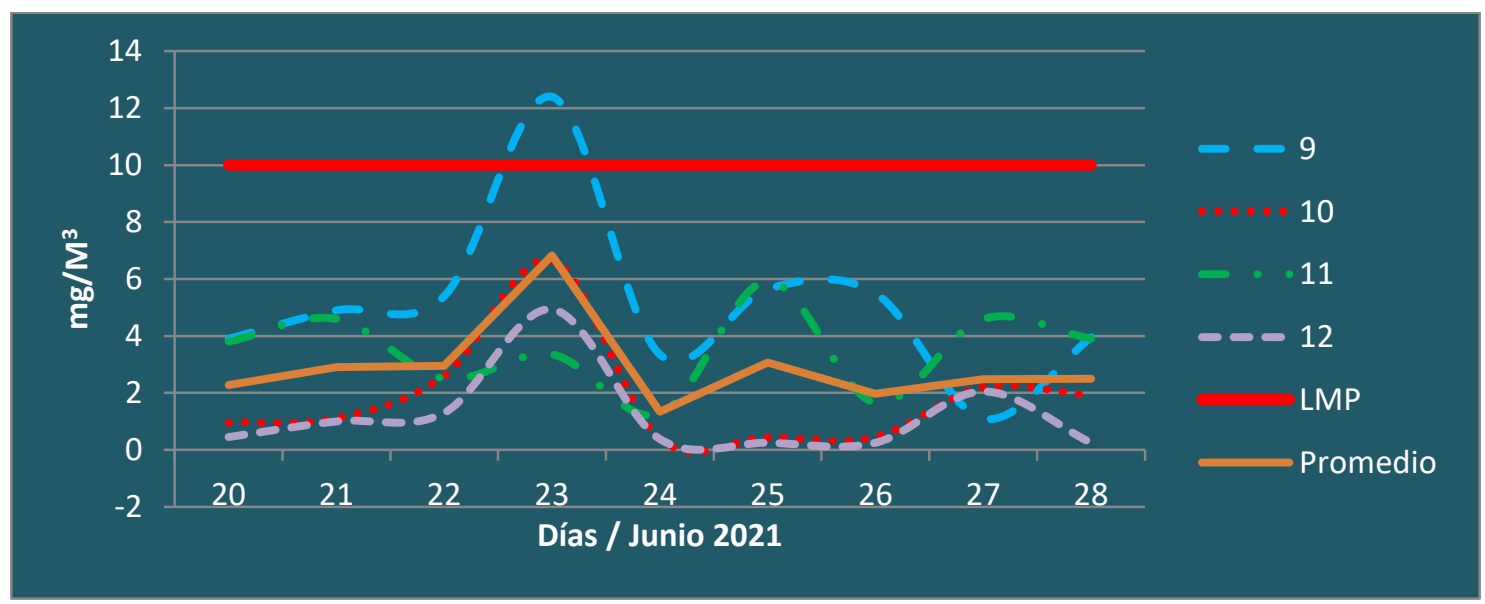

Figura 5: Comportamiento de los Niveles de Monóxido de Carbono en el distrito de Yarinacocha por la Fiesta de San Juan 2021 
UNIVERSIDAD NACIONAL DE UCAYALI

Revista de Investigación Universitaria

Versión electrónica 2664 - 8423

ARTICULO ORIGINAL
Vol. $11 \mathrm{~N}^{\circ} 2$, pp. 615 - 629, julio/diciembre 2021

Recibido 02/10/2021

Aceptado 18/12/2021

Publicado 30/12/2021
En la Figura 5, se aprecia el comportamiento de los Niveles de Monóxido de Carbono en el distrito de Yarinacocha por la Fiesta de San Juan 2021, los niveles máximos promedios de $6.833 \mathrm{mg} / \mathrm{m}^{3} \mathrm{CO}$ fueron obtenidos durante el día 23 de Junio, el pico máximo se analizó en el punto perteneciente al cruce Av. Miraflores y Av. Yarinacocha con un nivel de $12.4 \mathrm{mg} / \mathrm{m}^{3}$ CO superando el LMP de $10 \mathrm{mg} / \mathrm{m}^{3} \mathrm{CO}$ considerado en los Estándares de Calidad Ambiental para Aire del D.S.003-2017MINAM.

\section{Tabla 5}

Análisis de Varianza de los Niveles de Monóxido de Carbono según la etapa de la Fiesta de San Juan

\section{Comparaciones múltiples}

Variable dependiente: $\mathrm{CO}\left(\mathrm{mg} / \mathrm{M}^{3}\right)$

\begin{tabular}{llrrrrr} 
(I) Etapa & (J) Etapa & $\begin{array}{r}\text { Diferencia de } \\
\text { medias (I-J) }\end{array}$ & Error estándar & & Sig. & \multicolumn{2}{c}{ Intervalo de confianza al 95\% } \\
& & & & \multicolumn{1}{c}{$\begin{array}{c}\text { Límite } \\
\text { inferior }\end{array}$} & \multicolumn{1}{c}{$\begin{array}{c}\text { Límite } \\
\text { superior }\end{array}$} \\
\multirow{2}{*}{ Antes } & Durante & $-2,33944^{*}$ &, 63550 &, 001 & $-3,8503$ &,- 8286 \\
& Después &,- 22028 &, 63550 &, 936 & $-1,7311$ & 1,2906 \\
\multirow{2}{*}{ Durante } & Antes & $2,33944^{*}$ &, 63550 &, 001 &, 8286 & 3,8503 \\
& Después & $2,11917^{*}$ &, 63550 &, 003 &, 6083 & 3,6300 \\
\multirow{2}{*}{ Después } & Antes &, 22028 &, 63550 &, 936 & $-1,2906$ & 1,7311 \\
& Durante & $-2,11917^{*}$ &, 63550 &, 003 & $-3,6300$ &,- 6083
\end{tabular}

*. La diferencia de medias es significativa en el nivel 0.05 .

En la tabla 5, se puede apreciar que los niveles de Monóxido de Carbono antes y durante la fiesta de San Juan difieren entre sí con una significancia de 0.01 , siendo esta una diferencia de medias significativa; los niveles de Monóxido de Carbono durante y después de la fiesta difieren entre sí con una significancia de 0.03 , siendo esta una diferencia de medias significativa; en los niveles de Monóxido de Carbono antes y después de la fiesta de San Juan no difieren entre sí, con una significancia de 0.936 , siendo esta una diferencia de medias no significativa. 
Tabla 6

Análisis de Subconjuntos de los niveles de Monóxido de Carbono según la etapa de la Fiesta de San Juan

\begin{tabular}{lcrr}
\hline \multicolumn{4}{c}{ CO $\left(\mathbf{m g} / \mathbf{m}^{\mathbf{3}}\right)$} \\
\hline $\begin{array}{l}\text { HSD Tukey } \\
\text { Etapa }\end{array}$ & $\mathrm{N}$ & Subconjunto para alfa $=0.05$ & \\
& & 1 & \\
Antes & 36 & 2,8792 & \\
Después & 36 & 3,0994 & 5,2186 \\
Durante & 36 &, 936 & 1,000 \\
Sig. & & \\
Se visualizan las medias para los grupos en los subconjuntos homogéneos. \\
a. Utiliza el tamaño de la muestra de la media armónica $=36,000$.
\end{tabular}

En la tabla 6, se puede apreciar que los subconjuntos que corresponden a antes y después de la Fiesta de San Juan están agrupadas en el grupo " 1 " y el subgrupo que corresponde a los niveles de Monóxido de Carbono durante la Fiesta de San Juan está excluida en un grupo propio, aseverando que los niveles de Monóxido de Carbono Durante la Fiesta de San Juan son diferentes antes y después de esta, afirmando la hipótesis que la Fiesta de San Juan afecta negativamente los niveles de Monóxido de Carbono en la ciudad de Pucallpa del 2021.

\section{Conclusiones}

Los niveles de Monóxido de Carbono causado por la festividad de "San Juan" en la ciudad de Pucallpa en el 2021, que los Índices de Nacionales de Calidad de Aire fueron
"Buenos" antes y después de la festividad, pero de clasificación "Moderada" durante la fiesta.

Los niveles de Monóxido de Carbono antes de la festividad de "San Juan" fueron en promedio de $4.270 \mathrm{mg} / \mathrm{m}^{3}$, durante promedio $6.660 \mathrm{mg} / \mathrm{m}^{3}$ y después promedio de $4.940 \mathrm{mg} /$ $\mathrm{m}^{3}$ en la ciudad de Pucallpa en el año 2021.

8 de 10 familias usaron leña o carbón para la cocción del Juane y que 9 de 10 familias elaboraron o cocinaron el juane de la fiesta de “San Juan- Pucallpa 2021” el 23 de junio (un día antes de la Fiesta).

La actividad de la cocción del "Juane de Arroz" es una de las principales fuentes emisoras de Monóxido de Carbono durante la 
festividad de "San Juan" en la ciudad de Pucallpa en el año 2021.

\section{Referencia bibliográfica}

ANDINA. (19 de Junio de 2019). andina.pe.

Obtenido de

https://andina.pe/agencia/noticia-conoce-

como-celebra-amazonia-peruana-fiesta-

san-juan-755896.aspx

ATSDR. (2012). Resumen de Salud Pública:

Monóxido de Carbono. EE.UU.:

Departamento de salud y servicios humanos de los EE.UU., Servicio de Salud Pública. Obtenido de https://www.atsdr.cdc.gov/es/phs/es_phs2 01.pdf

Bautista Baca, J. (2018). "Evaluación de la concentración de metano, monóxido y dióxido de carbono en el aire, mediante tecnología de drones y hardware libre en la ciudad de Puerto Maldonado". Puerto Maldonado: Universidad Nacional Amazónica de Madre De Dios. http://repositorio.unamad.edu.pe/handle/U NAMAD/364

Canales Manchuria, G.-M. (2019). Monitoreo y evaluación de los gases monóxido de carbono (CO), dióxido de carbono (CO2), hidrogeno sulfurado (H2S) presentes en el distrito de Alto Selva Alegre - Arequipa.
Arequipa: Universidad Nacional de San Agustín de Arequipa. http://repositorio.unsa.edu.pe/handle/UNS $\mathrm{A} / 10265$

Canepa Koch, G., Hernandez Macedo, M., Biffi Isla, V., \& Zuleta Garcia, M. (2011). Cocina e identidad: La culinaria peruana como patrimonio cultural inmaterial (1 ed.). Lima: Ministra de Cultura.http://repositorio.cultura.gob.pe/bi tstream/handle/CULTURA/681/COCINA $\% 20 \mathrm{E} \%$ 20IDENTIDAD.pdf?sequence $=1$ \&isAllowed $=\mathrm{y}$

EPA. (28 de Septiembre de 2020). Agencia de Protección Ambiental de Estados Unidos. Obtenido de https://espanol.epa.gov/cai/impacto-delmonoxido-de-carbono-en-la-calidad-delaire-de-los-interiores

EPA. (30 de Marzo de 2021). Agencia de Protección Ambiental de Estados Unidos. Obtenido de https://espanol.epa.gov/espanol/el-humode-la-lena-y-su-salud

Flores Yucra, R. (2017). Determinación del nivel de contaminación de dióxido de carbono por parque automotor en la ciudad de Puno. Puno: Universidad Nacional Del Altiplano. Obtenido de http://repositorio.unap.edu.pe/bitstream/ha 
ndle/UNAP/9281/Flores_Yucra_Ruben.pd

$\mathrm{f}$ ?sequence $=1$ \&isAllowed $=\mathrm{y}$

Fonseca Adianzen, A. (2018). Niveles de monóxido de carbono en el aire de la ciudad de Tingo María, año 2017. Tingo Maria: Universidad Nacional Agraria de la Selva.

http://repositorio.unas.edu.pe/bitstream/ha ndle/UNAS/1494/ARFA_2018.pdf?seque nce $=1 \&$ is Allowed $=\mathrm{y}$

Gomez Castañeda, A., \& Gonzalez Yjuma, H. (2020). Determinación de la concentración de Monóxido de Carbono ( $\mathrm{CO}$ ) del parque automotor de las ciudades de Pucallpa y Aguaytía. Ucayali: Universidad Nacional de

Ucayali. https://alicia.concytec.gob.pe/vufind/Reco rd/RUNU_c5d85834cadabf80147e6cf645 $29 \mathrm{a} 454$

Gorritty, M., Torrico, T., \& Montenegro, Y. (2011). Determinación de la tasa de emisión de $\mathrm{CO}$ en cocinas mejoradas a leña con chimenea mediante el modelo de caja con ventilación constante. RevActaNova, 96-109. Obtenido de http://www.scielo.org.bo/scielo.php?script $=$ sci_arttext $\&$ pid $=$ S1683-

07892011000100005

Instituto Nacional de Cultura. (2010).

Resolución Directoral Nacional N575-
INC. Lima: Ministerio de Cultura. http://administrativos.cultura.gob.pe/intran et/dpcn/anexos/80_1.pdf?18877

MINAM. (2016). R.M.181-2016MINAM/INDICE DE CALIDAD DEL AIRE. Lima: El Peruano. https://www.minam.gob.pe/wpcontent/uploads/2016/07/RM-N\%c2\%b0181-2016-MINAM.pdf MINAM. (2019). Protocolo Nacional de Monitoreo de la Calidad Ambiental del Aire. Lima: El Peruano. https://sinia.minam.gob.pe/download/file/f id/65578

Nige, B., Perez-Padilla, R., \& Albalak, R. (2000). Indoor air pollution in developing countries: a major environmental and public health challenge. Bulletin of the World Health Organization, 78(9), 10781092.

https://www.ncbi.nlm.nih.gov/pmc/articles /PMC2560841/pdf/11019457.pdf

Pérez-Padilla, J., Regalado-Pineda, J., \& Morán-Mendoza, A. (1999). La inhalación doméstica del humo de leña y otros materiales biológicos. Un riesgo para el desarrollo de enfermedades respiratorias. Gac. Méd. Méx, 135(1),19-30. http://www.anmm.org.mx/bgmm/1864_20 07/1999-135-1-19-30.pdf 
UNIVERSIDAD NACIONAL DE UCAYALI

Revista de Investigación Universitaria

Versión electrónica 2664 - 8423

ARTICULO ORIGINAL
Vol. $11 \mathrm{~N}^{\circ}$ 2, pp. $615-629$, julio/diciembre 2021

Recibido 02/10/2021

Aceptado 18/12/2021

Publicado 30/12/2021
Perú21. (24 de Junio de 2017). peru21.pe.

Obtenido

de

https://peru21.pe/cultura/fiesta-san-juan-

conoce-historia-variedades-juane-83330-

noticia/

Public Health Madison \& Dane County. (Febrero de 2004). Contaminación por humo de leña. Obtenido de https://www.publichealthmdc.com/espanol /salud-ambiental/calidad-del-aire/calidaddel-aire-exterior/contaminacion-por

Smith, K. (2006). El uso doméstico de leña en los países en desarrollo y sus repercusiones en la salud. Los bosques y la salud humana,

$57(2)$

$1-5$.

http://www.fao.org/3/a0789s/a0789s09.ht

$\mathrm{m}$

Torres-Duque, C., García-Rodriguez, M., \& González-García, M. (2016). Enfermedad pulmonar obstructiva crónica por humo de leña: ¿un fenotipo diferente o una entidad distinta? ArchBronconeumol, 52(8), $425-$ 431.

https://www.archbronconeumol.org/espdf-S0300289616300655 